\title{
Influenza Among Breast Cancer Survivors in South Korea: A Nationwide Population-Based Study
}

\author{
JAESUNG HEO $^{1}$, MISON CHUN ${ }^{1}$, YOUNG-TAEK OH ${ }^{1}, \mathrm{O} \mathrm{KYU} \mathrm{NOH}^{1,2}$ and LOGYOUNG KIM ${ }^{3}$ \\ ${ }^{1}$ Department of Radiation Oncology, Ajou University School of Medicine, Suwon, Republic of Korea; \\ ${ }^{2}$ Office of Biostatistics, Ajou University School of Medicine, Suwon, Republic of Korea; \\ ${ }^{3}$ Health Insurance Review and Assessment Service, Seoul, Republic of Korea
}

\begin{abstract}
Background/Aim: In this study, we analyzed the prevalence of influenza disease in breast cancer survivors using claims data from the Health Insurance Review and Assessment Service (HIRA) in South Korea. Patients and Methods: We defined influenza among a nationwide cohort of 87,843 patients who were diagnosed with breast cancer and underwent surgery from January 1, 2010 to December 31, 2014, based on HIRA claim data. Descriptive statistics were calculated to estimate the frequency of influenza disease using diagnostic code and utilization pattern at medical institutions. Results: Two thousand four hundred breast cancer survivors $(2.7 \%)$ were diagnosed with influenza. The overall frequency of influenza increased from November $(n=98)$ and peaked in February $(n=1,745)$. Over $60 \%$ $(2,693)$ of claims for influenza disease treatment were in the clinic, whereas general hospitals accounted for $22.0 \%$ (873). Among 3,967 claims, admission rate was $7.6 \%(n=301)$ and the average length of hospitalization was 4.7 days. Elderly breast cancer survivors over 70 years old had the longest length of hospitalization at 5.9 days. Conclusion: Breast cancer survivors are more susceptible to influenza than non-cancer survivors. It is important not only to raise the vaccination rate among young cancer survivors, but also to quickly identify symptoms and begin treatment for flu in elderly cancer survivors.
\end{abstract}

Worldwide, breast cancer is the most commonly diagnosed malignancy among women (1). Especially in traditionally low-risk areas, where changes in lifestyle have been significant, breast cancer incidence rates have nearly doubled in recent decades (2). In South Korea, the number of patients

This article is freely accessible online.

Correspondence to: Mison Chun, Department of Radiation Oncology, Ajou University School of Medicine, 206 Worldcup-ro, Yeongtong-gu, Suwon 443-721, Republic of Korea. Tel: +82 312195885, Fax: +82 312195894, e-mail: chunm@ajou.ac.kr

Key Words: Influenza, breast cancer, pulmonary disease, admission. diagnosed with breast cancer has rapidly increased, reaching 12,206 in 2012, which was 3-fold higher than 10 years earlier (3). As the number of breast cancer survivors has increased, managing quality of life had become increasingly important over time (4).

Influenza infection is a major cause of acute respiratory disease. Influenza epidemics are associated with economic burden to medical finance, in addition to mortality (5). Those with medical conditions such as chronic disease, metabolic syndrome, or underlying malignancy are susceptible to influenza infection (6). In particular, cancer survivors could experience reduced immunity due to chemotherapy, disease, or medications such as corticosteroids (7). To prevent influenza infection, annual vaccination for influenza is recommended for cancer survivors (8).

However, despite recommendations there are some cancer survivors who tend to be reluctant to undergo vaccination (9). These attitudes towards vaccination were attributed to anxiety to complication after vaccination, lack of awareness of necessity, and lack of proper advice by medical staff (10). In a previous study, the influenza vaccination rates for cancer survivors and non-cancer survivors were $50.3 \%$ and $31.5 \%$, respectively, in South Korea (11). However, vaccination for influenza varies based on age. More than $75 \%$ of people over 65 years old were vaccinated and only $26 \%$ of cancer survivors aged 19-44 years were vaccinated (12).

Although studies have examined influenza vaccination rates among cancer survivors in South Korea, there are no studies about the prevalence of influenza disease among cancer survivors based on nationwide data. In this study, we analyzed the prevalence of influenza disease among breast cancer survivors using claims data from the Health Insurance Review and Assessment Service (HIRA) in South Korea.

\section{Patients and Methods}

South Korea has the National Health Insurance (NHI) system and every resident in South Korea is obligated to join NHI. Claim data in HIRA were generated during patient visits to medical institutions. Based on NHI, the HIRA database consisted of four subgroup 
tables. One is a general specification table and included patient demographics (patient gender, age, residential area), institution characteristic (location, department, admission), and diagnosis. The second table is about medical history and contained procedures and medications from hospitalization. In addition to the main diagnosis, we could search additional diagnoses of patients in the third diagnosis table. The fourth table included information about drugs prescribed outside of the hospital. In this study, we defined the nationwide cohort as patients who were diagnosed with breast cancer and underwent surgery from January 1, 2010 to December 31, 2014 based on HIRA claim data. The institutional review board (IRB) of the Ajou University Hospital approved the study (IRB No. AJIRB-MED-EXP-15-538).

The study population consisted of patients who had a cancer diagnosis of category C50 based on the Korean Classification of Disease, sixth edition (KCD-6), a version of the International Classification of Disease 10 (ICD-10), modified for the Korean health care system. Subgroups of invasive breast cancer included nipple and areola (C500), central portion of the breast (C501), upper-inner quadrant of the breast (C502), lower-inner quadrant of the breast (C503), upper-outer quadrant of the breast (C504), lowerouter quadrant of the breast (C505), axillary tail of the breast (C506), overlapping lesion of the breast (C508) and breasts, unspecified (C509). We also used procedure codes: N7131, simple mastectomy; N7133, partial mastectomy; and N7135, radical mastectomy.

Follow-up data for breast cancer survivors were collected for 6 years, from January 2010 to June 2015 . To confirm breast cancer survivors with influenza disease, we used diagnostic codes in the medical claim dataset associated with influenza disease (including all hospital admissions, medical office visits, and emergency room visits), using ICD-10 as the principal diagnosis. Influenza is represented as J9 (Influenza due to specific identified influenza virus), J10 (Influenza due to other identified influenza virus), and J11 (Influenza, virus not identified). Regional classification was performed using local area identifiers.

We analyzed the prevalence of influenza infection after diagnosis with breast cancer. Additionally, we evaluated characteristics of breast cancer survivors with influenza according to age. To analyze the patient's utilization patterns of medical institutions, we classified hospitals into four categories (general hospital, hospital, private clinic, and public health center) with specific medical department categories. We also analyzed differences in hospitalization between each age group. All statistical analysis was performed with R 3.0.2.

\section{Results}

A total of 87,843 breast cancer survivors who were diagnosed with invasive breast cancer and received surgery between 2010 and 2014 were identified based on HIRA claim data. The median age at diagnosis for patients with breast cancer was 50 years, and 49 years for breast cancer survivors diagnosed with influenza. The frequency of influenza among breast cancer survivors is shown in Table I. The total claim data of breast cancer survivors who visited a hospital with influenza was 3,960 . The overall prevalence of influenza in breast cancer survivors was $2.7 \%(\mathrm{n}=2,400)$. In analysis based on age, breast cancer survivors with influenza were most likely to be 40 to 49 years of age (34\%).
Table I. The frequency of influenza in breast cancer survivors $(N=2,400)$.

\begin{tabular}{lcc}
\hline Age & Breast cancer survivors & Influenza (\%) \\
\hline $10-39$ & 11,124 & $454(4.1)$ \\
$40-49$ & 31,600 & $808(2.6)$ \\
$50-59$ & 26,720 & $715(2.7)$ \\
$60-69$ & 12,305 & $292(2.4)$ \\
$70-99$ & 6,093 & $131(2.2)$ \\
\hline Total & 87,842 & $2,400(2.7)$ \\
\hline
\end{tabular}

Considering prevalence based on each age group population, young age group between 10 and 39 was most common $4.1 \%(\mathrm{n}=454)$ (Table I).

Monthly disease incidence based on claim data showed a seasonal change (Figure 1). Incidence increased from November $(\mathrm{n}=98)$ and showed the peak in February $(n=1,745)$. Overall, there were 3,967 claims for influenza disease. Over $60 \%(2,693)$ of claims for influenza disease treatment were in the clinic, whereas general hospitals accounted for $22.0 \%$ (873) of all claims (Figure 2A). Differences by region and type of medical institution were seen in the influenza data (Figure 2B). In urban areas, the proportion of general hospital use was relatively high, at over $25 \%$. On the other hand, in rural areas, the proportion of general hospital use was $19.3 \%$. Also, claims by public health centers in local areas was confirmed to be over $2 \%$ of total claims.

We compared characteristics between the inpatient and outpatient settings with influenza disease. Among 3,967 claims, claim for admission was $7.6 \%(n=301)$ (Table II). Among the outpatients, the 40 s were most frequent (34.4\%) in the breast cancer survivors. Of the inpatients, the $50 \mathrm{~s}$ were most common and the proportion of the elderly over 60 years old was increased compared to the outpatient setting. Analyzing the admission rate by age group, the rate of admission in the elderly group over 70 years old was the highest (12.0\%) (Figure 3A). On the other hand, the rate of admission in the age group between 40 and 49 was the lowest $(6.4 \%)$. The average length of hospitalization in elderly breast cancer survivors over 70 years old increased up to 5.9 days, though it was 4.7 days in the population as a whole (Figure 3B).

\section{Discussion}

Seasonal influenza during autumn and winter is a major cause of acute respiratory disease (13). In particular, cancer survivors are more susceptible to influenza than non-cancer survivors owing to their low immunity. Therefore, 




Figure 1. Monthly diagnosis of influenza (2010-2015).

vaccination is the most effective method to prevent influenza infection. In South Korea, a previous study reported that more than $50 \%$ of cancer survivors received influenza vaccination, a higher rate than that in non-cancer survivors (12). The proportion of vaccination in breast cancer survivors was more than other cancer types, such as stomach, liver, and colon cancer (11).

In this study, we analyzed the frequency of influenza in breast cancer survivors. The prevalence rate of influenza in breast cancer survivors was $2.7 \%(n=2400)$, higher than in the whole population, where prevalence rate was $1.6 \%$ $(\mathrm{n}=825,803)$ (14). Cancer survivors belonged to a more vulnerable group for influenza than non-cancer survivors. Therefore, promotion of vaccination and prevention of influenza through lifestyle change is important. In the United States, a new approach named "Healthy People 2020 Goals" had a goal of $80-90 \%$ vaccination coverage among the target population (15). To prevent influenza infection, cancer survivors need a lifestyle that includes covering with a handkerchief when coughing, or washing hands after going out.

There was a disparity in the prevalence of influenza by age groups. Young breast cancer survivors (age group between 10 and 39 years) had a higher prevalence of influenza than elderly age group over 70 years old. Although in general elderly people had greater comorbidity
Table II. Distribution of claim data for influenza by age group according to admission $(N=3,967)$.

\begin{tabular}{cccccc}
\hline & \multicolumn{2}{c}{ Outpatient } & & \multicolumn{2}{c}{ Inpatient } \\
\cline { 2 - 3 } \cline { 5 - 6 } & No & $\%$ & & No & $\%$ \\
\hline $10-39$ & 608 & 16.6 & & 47 & 15.6 \\
$40-49$ & 1,262 & 34.4 & & 87 & 28.9 \\
$50-59$ & 1,098 & 30.0 & & 97 & 32.2 \\
$60-69$ & 463 & 12.6 & & 38 & 12.6 \\
$70-99$ & 235 & 6.4 & & 32 & 10.6 \\
\hline Total & 3,666 & 100 & & 301 & 100 \\
\hline
\end{tabular}

and reduced immune status than young people, our results confirmed that those in the young age group were more susceptible to influenza. Previous studies confirmed that there was a difference in vaccination rate between age groups, and young age groups exhibited lower influenza vaccination than elderly groups (9). In South Korea, free influenza vaccination was restrictively offered to infants and elderly people older than 65 years of age. To improve vaccination rates for young cancer survivors, additional free 
A

3000

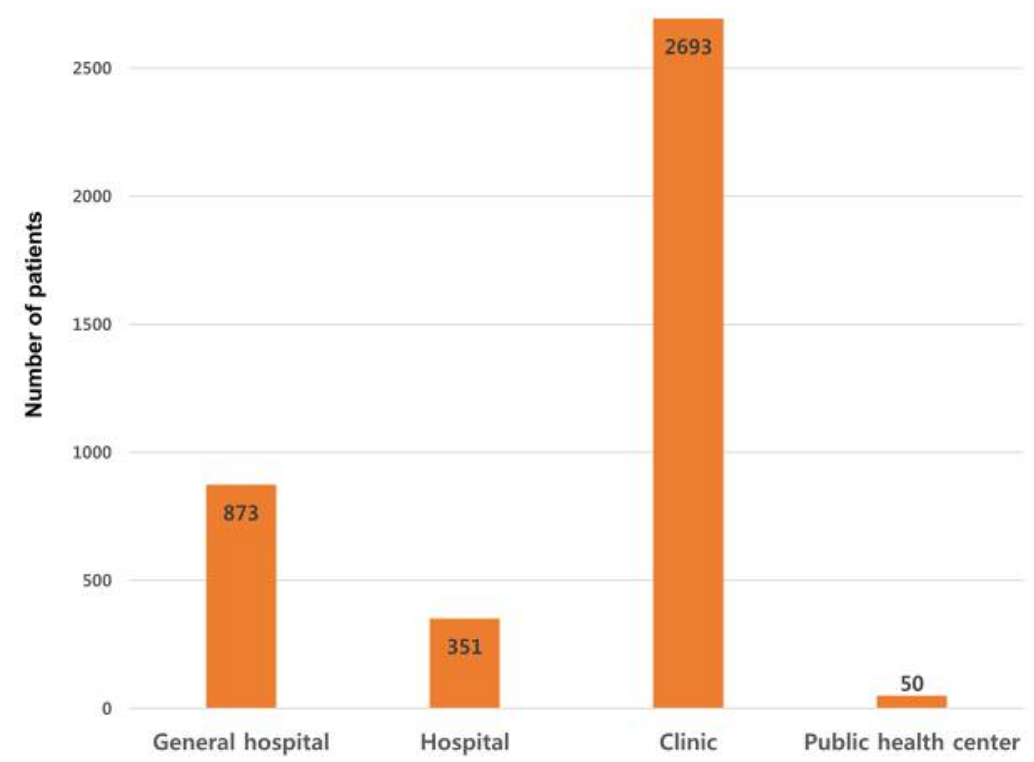

B

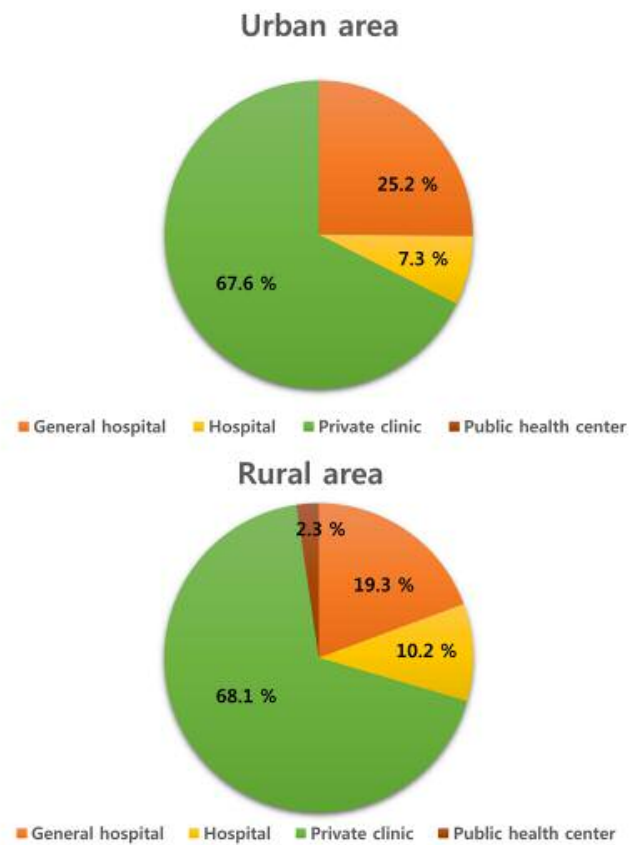

Figure 2. A) Frequency of influenza diagnosis by medical institution. B) The proportion of medical institution utilization based on region.

A

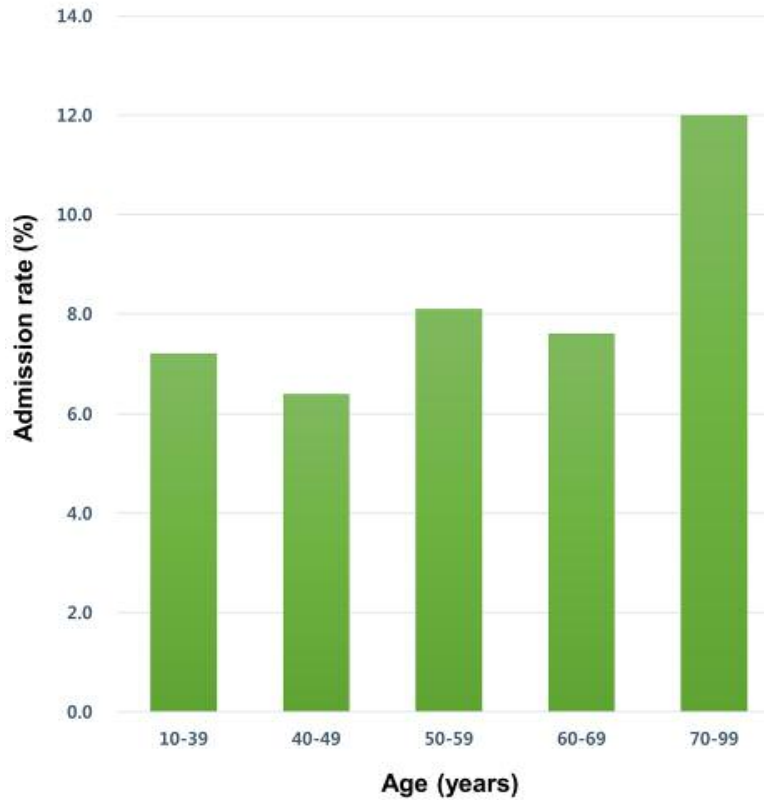

B



Figure 3. A) Average hospital admission rate by age group. B) Mean hospitalization length by age group. 
vaccination coverage for young breast cancer survivors should be considered. Also, mass media such as television programs, newspapers, and wire service articles were used to promote vaccination in the United States (16). This study showed positive associations for campaigns using mass media to earlier vaccination timing and increased vaccination rate. Recently, a campaign leveraging social media platforms such as Facebook, YouTube, and blogs was used to induce behavioral changes (17). An increased influenza vaccination rate was related to a reduction in hospital admissions and a decrease in hospitalization cost for cancer survivors (18).

We analyzed the frequency pattern of influenza according to time. The frequency of influenza disease in breast cancer survivors increased from November with the peak confirmed at February. Therefore, early vaccination taking into account likely epidemic periods is important. Additionally, because breast cancer survivors received chemotherapy (CT) or radiotherapy (RT) before or after surgery, it is important to determine the appropriate timing of vaccination. Generally, it is recommended that vaccination should be completed at least 1 month before initiation of $\mathrm{CT}$ and RT, or 1 month after CT and RT (8). Therefore, coordination between oncology specialists and primary care providers is an essential component of cancer survivorship. Claire et al. confirmed that cancer survivors who visited a cancer specialist and a primary physician were most likely to receive good medical service, such as vaccination and periodic follow-up (9).

In this study, we also showed that influenza was most frequently diagnosed in local clinics (Figure 2A). However, primary care providers lack the knowledge and experience to manage cancer survivors. Cancer survivors who were treated by only primary physicians in local clinics, were less likely to undergo secondary cancer screening and surveillance (19). Therefore, educational programs and guidelines reflecting the healthcare environment in South Korea are necessary for primary physicians. The utilization of public health centers for treatment was high in rural areas that had limited medical access based on geographical barriers such as island and mountain environments (Figure 2B). Therefore, areas with low medical access need not only medical help but also social-economic support for cancer survivorship management by public health centers.

Elderly breast cancer survivors had a higher vaccination rate and a lower frequency of medical institution visits than young breast cancer survivors (11). However, after diagnosis with influenza, disease status of elderly cancer survivors tended to be more severe than young cancer survivors. Among all age groups, the admission rate in the elderly group over 70 years of age was highest $(12 \%)$ and the average length of hospitalization was over five days. Therefore, besides prevention by vaccination, early detection of influenza-related symptoms such as high fever, headache, and muscle pain was important for elderly cancer survivors. Additionally, elderly people among breast cancer survivors had a tendency to have several comorbidities (20). These comorbidities could decrease immunity and increase disease severity. When approached from an economic point of view, the cost of hospitalization per day in South Korea was over $\$ 500$ and it could put a burden on medical finances and an individual's economic status (21). Therefore, to reduce the hospitalization period as much as possible, elderly cancer survivors must be screened and monitored closely for potential symptom.

There were a few limitations to this study. First, we confirmed influenza disease based on claims data from HIRA, which offered only code and demographic information. Clinical data related to severity of influenza disease such as fever, symptoms, and laboratory tests at medical institutions were not available from HIRA. Additionally, factors associated with severity of breast cancer, such as stage and pathologic report, were not confirmed. Therefore, future studies combining HIRA claim data with clinical datasets such as national health insurance service data are necessary. Second, we did not examine for variation over seasonal influenza. The degree of severity varied according to type of epidemic. These differences could affect the diagnosis and admission rate of influenza disease. Third, we did not confirm whether the breast cancer survivors were vaccinated against influenza. Because vaccination for influenza was recognized as non-payment, claim data by HIRA, which could check only payment in hospital, did not involve immunization status of breast cancer survivors. Therefore, we could not determine the correlation between vaccination and influenza disease. Future studies are needed to demonstrate these direct effects of vaccination.

\section{Conclusion}

Influenza disease had an association with risk to public health and economic burden to medical finance. Owing to lower immune status, cancer survivors are at high-risk for respiratory disease caused by influenza virus. Therefore, annual vaccination for influenza is recommended for cancer survivors before the onset of the flu season. For young breast cancer survivors, to increase vaccination rates, additional free vaccination coverage and education using social and mass media are needed. In elderly cancer survivors with high severity of disease, early detection of influenza-related symptoms and quick treatment is necessary. Ultimately, through coordination between oncology specialists and primary care physicians, systematic management for influenza disease in breast cancer survivors is needed. 


\section{Conflicts of Interest}

The Authors report no conflicts of interest.

\section{References}

1 Siegel RL, Miller KD and Jemal A: Cancer statistics, 2016. CA Cancer J Clin 66(1): 7-30, 2016.

2 Seow A, Duffy SW, McGee MA, Lee J and Lee HP: Breast cancer in singapore: Trends in incidence 1968-1992. Int J Epidemiol 25(1): 40-45, 1996.

3 Oh C-M, Won Y-J, Jung K-W, Kong H-J, Cho H, Lee J-K, Lee $\mathrm{DH}$ and Lee KH: Cancer statistics in korea: Incidence, mortality, survival, and prevalence in 2013. Cancer Res Treat 48(2): 436450, 2016.

4 Kim SH, Cho YU and Kim SJ: Weight gain and its correlates among breast cancer survivors. Asian Nursing Res 7(4): 161167,2013

5 Stafford KA, Sorkin JD and Steinberger EK: Influenza vaccination among cancer survivors: Disparities in prevalence between blacks and whites. J Cancer Surviv: research and practice 7(2): 183-190, 2013.

6 Pedrazzoli P, Baldanti F, Donatelli I, Castrucci MR, Puglisi F, Silvestris $\mathrm{N}$ and Cinieri S: Vaccination for seasonal influenza in patients with cancer: Recommendations of the italian society of medical oncology (aiom). Ann Oncol 25(6): 1243-1247, 2014.

7 Wingard JR: Influenza: Preparedness for an inevitable "emergency" for oncology and bmt units. J Natl Compr Canc Netw 6(2): 215-222, 2008.

8 Rubin LG, Levin MJ, Ljungman P, Davies EG, Avery R, Tomblyn M, Bousvaros A, Dhanireddy S, Sung L, Keyserling H and Kang I: 2013 idsa clinical practice guideline for vaccination of the immunocompromised host. Clin Infect Dis 58(3): e44100,2014

9 Snyder CF, Frick KD, Peairs KS, Kantsiper ME, Herbert RJ, Blackford AL, Wolff AC and Earle CC: Comparing care for breast cancer survivors to non-cancer controls: A five-year longitudinal study. J Gen Intern Med 24(4): 469-474, 2009.

10 Shin DW, Sunwoo S and Lee J: Management of cancer survivors in korea. J Korean Med Assoc 58(3): 216-226, 2015.

11 Choi K-H, Park SM, Lee K, Lee JH and Park J-S: Influenza vaccination and associated factors among korean cancer survivors : A cross-sectional analysis of the fourth \& fifth korea national health and nutrition examination surveys. J Korean MedSci 29(8): 1061-1068, 2014.
12 Oh MG, Han MA, Yun NR, Park J, Ryu SY, Kim DM and Choi SW: A population-based, nationwide cross-sectional study on influenza vaccination status among cancer survivors in korea. Int J Environ Res Public Health 12(8): 10133-10149, 2015.

13 Liu T, Li Z, Zhang S, Song S, Julong W, Lin Y, Guo N, Xing C, $\mathrm{Xu} \mathrm{A}, \mathrm{Bi} \mathrm{Z}$ and Wang $\mathrm{X}$ : Viral etiology of acute respiratory tract infections in hospitalized children and adults in shandong province, china. Virol J 12: 168, 2015.

142014 National Health Insurance Statistical Yearbook, Seoul, South Korea, 2015.

15 Healthy People 2020 (Available from: https://www.healthy people.gov/).

16 Yoo BK: How to improve influenza vaccination rates in the u.S. J Prev Med Public Health 44(4): 141-148, 2011.

17 Heo J, Chun M, Lee HW and Woo JH: Social media use for cancer education at a community-based cancer center in South Korea. J Cancer Educ, 2016. doi:10.1007/s13187-016-1149-4 [Epub ahead of print]

18 Lin HH, Hsu KL, Ko WW, Yang YC, Chang YW, Yu MC and Chen KT: Cost-effectiveness of influenza immunization in adult cancer patients in taiwan. Clin Microbiol Infect 16(6): 663-670, 2010.

19 Shin DW, Kim Y, Baek YJ, Mo HN, Choi JY and Cho J: Oncologists experience with second primary cancer screening: Current practices and barriers and potential solutions. Asian Pac J Cancer Prev 13(2): 671-676, 2012.

20 Lee JE, Shin DW, Lee H, Son KY, Kim WJ, Suh YS, Kong SH, Lee HJ, Cho B and Yang HK: One-year experience managing a cancer survivorship clinic using a shared-care model for gastric cancer survivors in korea. J Korean Med Sci 31(6): 859-865, 2016.

21 Lim JK, Kim TH, Kilgore PE, Aiello AE, Choi BM, Lee KC, Yoo KH, Song Y-H and Kim Y-K: The association between influenza treatment and hospitalization-associated outcomes among korean children with laboratory-confirmed influenza. J Korean Med Sci 29(4): 485-493, 2014. 